\title{
A study of size effect in micro-forming with micro-hardness tests
}

\author{
Fuh-Kuo Chen ${ }^{*}$, Jia-Wen Tsai ${ }^{1}$ \\ Department of Mechanical Engineering, National Taiwan University, Taipei, Taiwan, ROC
}

\begin{abstract}
The size effect on the material properties in the micro-forming is examined in the present study using both the micro-hardness test and the finite element analysis. The measured hardness indicates that the hardness decreases as the dimension of the specimen is downscaled to a small size. The size effect on the material hardness is thus clearly observed. The good agreement between the measured hardness and the flow stress also confirms that the theoretical proportionality between the hardness and the flow stress is still valid in a specimen with downscaled dimensions. It suggests that the stress-strain relations of a downsized specimen can be constructed from the micro-hardness tests in conjunction with the compression tests, if the accurate data acquisition is not available in the compression test equipment for a downscaled specimen.
\end{abstract}

(C) 2006 Elsevier B.V. All rights reserved.

Keywords: Size effect; Micro-forming; Micro-hardness test; Flow stress; Finite element analysis

\section{Introduction}

Forming of micro-parts has drawn much attention from both the industrial practice and the academic research in the metal forming field. In addition to the technology developed for the manufacturing process, research efforts have also focused on the study of the variation of the mechanical properties when the dimension of the material is downscaled to a much smaller scale, which is called size effect. The material properties tests, such as tensile test and compression test, are necessary and fundamental in the study of size effect. However, the tests are getting more difficult to conduct when the material dimension is downsized to a very small scale. A specific testing machine with suitable load cells and accurate data acquisition apparatus is required to obtain the stress and strain relations for a downsized specimen. An alternative to obtain the material properties is the hardness test in which a special tension or compression testing machine with precision data acquisition is not mandatory to obtain the material properties of a downscaled specimen.

Geiger et al. [1], Kals and Eckstein [2] have conducted compression tests, tension tests, and bending tests, respectively, to study the variation of the material properties due to size effect. Literatures $[3,4]$ regarding the theory of plasticity have shown that the hardness is proportional to the flow stress by a factor of 3. However, the size effect on material hardness has not been

\footnotetext{
* Corresponding author. Tel.: +8862 23661322; fax: +886223631755.

E-mail address: fkchen@ntu.edu.tw (F.-K. Chen).

${ }^{1}$ Graduate student.
}

well studied and the validity of the theoretical proportionality between hardness and flow stress for the downsized specimens still remains uncertainty.

In the present study, the size effect on the hardness variation was studied with the use of the micro-hardness tests. Due to the inhomogeneous deformation in a compressed specimen, the finite element simulations were first preformed to identify the proper locations for the hardness measurement. The size effect on the material hardness was then studied.

\section{Compression tests}

In the present study, the compression tests were conducted using brass $(\mathrm{Cu}-\mathrm{Zn}$ alloy) as the specimen material. The downsized specimen of $5 \mathrm{~mm}$ in diameter and $7.5 \mathrm{~mm}$ in height was adopted for the compression tests. For the purpose of examining the size effect, a smaller size of specimen of $2 \mathrm{~mm}$ in diameter and $3 \mathrm{~mm}$ in height was also prepared for the compression tests. Both sizes of specimens were annealed at different temperatures of 500,600 , and $700{ }^{\circ} \mathrm{C}$, resulting in different grain sizes of 16,29 , and $74 \mu \mathrm{m}$, respectively. The stress-strain relations of both sizes of specimens with grain size of $29 \mu \mathrm{m}$ are shown in Fig. 1. It is noted in Fig. 1 that the flow stress becomes smaller as the specimen size is downscaled and the size effect is clearly observed. The effect of the grain size on the flow stress is also examined and the test results indicate that the specimen with a smaller grain size has a larger flow stress as predicted by the Hall-Petch equation. However, the difference is insignificant for the range of grain sizes examined in the present study. 


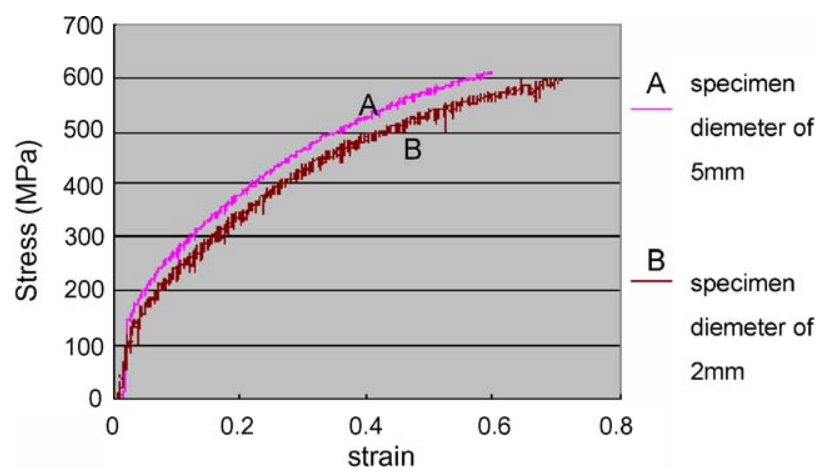

Fig. 1. Stress-strain curves for specimens with various diameters (grain size of $29 \mu \mathrm{m})$.

\section{Finite element analysis}

The 3D finite element program DEFORM was employed to determine the proper locations for hardness measurement where the effective strain and the effective stress are the same as the average strain and average stress in the compression tests. The finite element simulations were performed with different values of constant shear friction factor $(\mathrm{m})$ ranging from 0.1 to 0.4 to examine the effect of friction on the locations of hardness measurement. Only a quarter of the specimen was simulated in a 3D finite element model due to symmetric condition. In order to determine the proper locations, the effective strain and effective stress obtained from the finite element simulations in the cross-sections located at different heights of the deformed specimen, as shown in Fig. 2, were compared with the average stress and average strain of the whole specimen. The value of $h$ shown in Fig. 2 is the ratio of the height of the cross-section to the height of the deformed specimen $(H)$. On each cross-section, the distance from the center to the edge is divided into 15 equal intervals marked by $1-15$.

Fig. 3 shows the effective stress distributions on various crosssections when the specimen is deformed to $0.5 H_{0}$ with friction

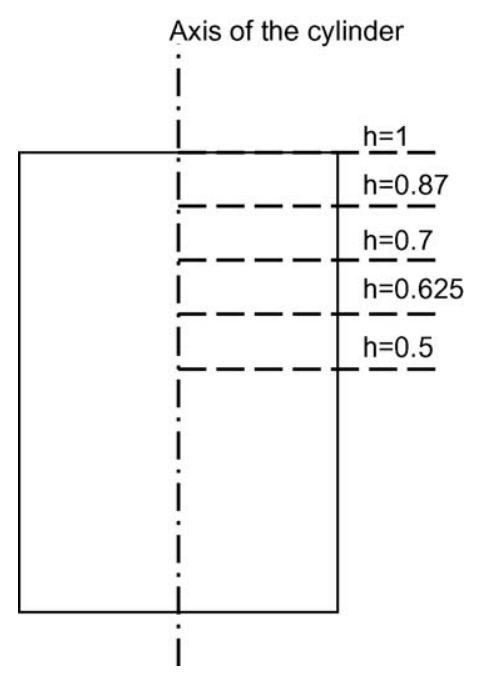

Fig. 2. The plane of analysis and the height ratios of the cross-sections.

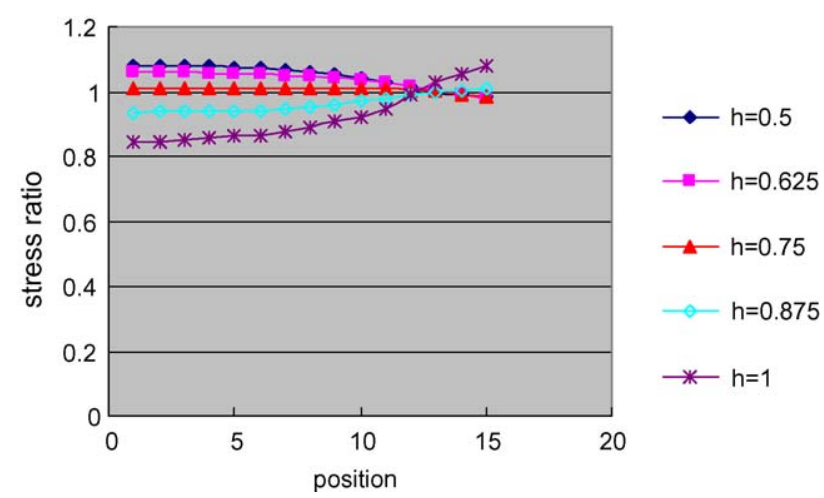

Fig. 3. Effective stress distributions in the deformed specimen $\left(H=0.5 H_{0}\right.$; $m=0.2$ ).

factors of $m=0.2$, where $H_{0}$ is the original specimen height. The stress ratio, which is defined as the ratio of effective stress at the point to the average stress of the whole specimen, is used as the ordinate, and the abscissa is the position from the center to the edge as mentioned above. As seen in Fig. 3, the effective stress increases from the top surface $(h=1)$ to the mid crosssection $(h=0.5)$ in most area on the cross-sections except near the edge of the cross-section. It is also noted that the effective stress on the cross-section of $h=0.75$ is evenly distributed and close to the average stress. However, the effective stress on various cross-sections converges to the average stress at the location about seven eighth of the radius away from the center. This location is then considered as the proper location for the hardness measurement.

\section{Micro-hardness tests}

In the present study, the micro-hardness test was performed to measure the hardness of a deformed specimen in a downscaled size using the AKASHI MVK-E micro-hardness tester. The micro-hardness test is improved from the Vickers hardness test, which is more suitable for measuring the hardness of a specimen in a small scale. Experimental load of $10 \mathrm{~g}$ was adopted in the micro-hardness test and the indented area was observed and measured by a high-resolution microscope. The hardness is measured for each deformed specimen with an instantaneous deformed height ranging from $0.5 \mathrm{H}_{0}$ to $0.9 \mathrm{H}_{0}$, where $H_{0}$ is the initial specimen height. Since there were specimens in two different dimensions and in three different grain sizes, six compression tests were required to perform for each specific deformed height. The deformed specimens were wire cut to the cross-sections determined from the finite element simulations for hardness measurement and the hardness was measured at the selected locations on each cross-section.

\section{Results and discussions}

The hardness distributions on various cross-sections at different instantaneous deforming heights for each kind of specimen were obtained from the micro-hardness tests. Fig. 4 shows the hardness distributions for the downscaled specimen of $2 \mathrm{~mm}$ in 


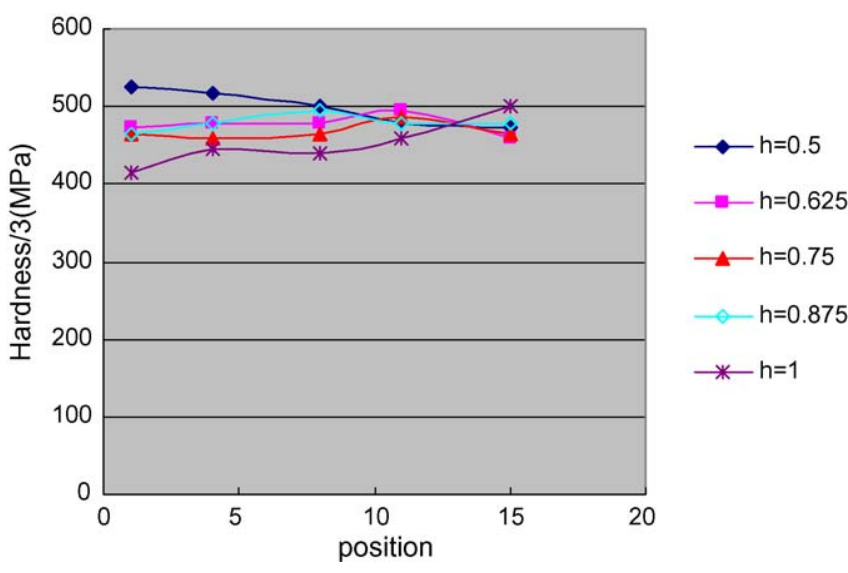

Fig. 4. Hardness distributions on the cross-sections of various specimen heights (specimen diameter $=2 \mathrm{~mm}$, grain size $=29 \mu \mathrm{m}, H=0.65 H_{0}$ ).

diameter and $29 \mu \mathrm{m}$ in grain size deformed to a height of $0.65 \mathrm{H}_{0}$. It is noted in Fig. 4 that the hardness distribution is a little scatter. The scattered data may attribute to the errors in the measurement of the indented area in the micro-hardness tests. Fig. 4 also indicates that the hardness distribution on the cross-section of $h=0.75$ is more uniform and the hardness distributions on various cross-sections converge to the same value at the location about seven eighth of the radius away from the center, which is consistent with the results predicted by the finite element simulations. The similar trend is also observed in other specimens. Hence, the proper location for the hardness measurement is confirmed.

Fig. 5 shows the measured hardness at various instantaneous deforming heights of the downscaled specimens with different grain sizes. It is barely observed that the specimen with smaller grain size has higher hardness. However, the difference is insignificant, which is similar to that obtained in the compression tests. The size effect on the hardness due to the downscaled dimension of specimen is shown in Fig. 6, in which the hardness measured from the specimens with the same grain size but different specimen dimensions is plotted. As seen in Fig. 6, the downscaled specimen has lower hardness than that of the larger

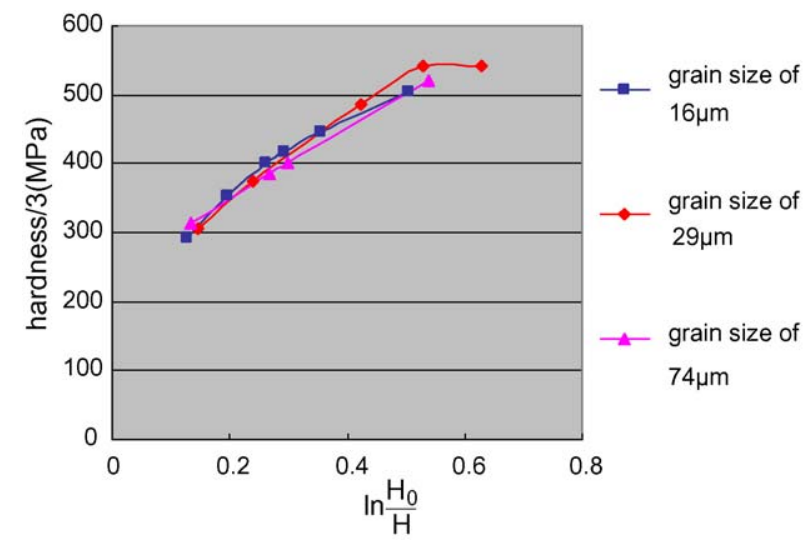

Fig. 5. Measured hardness at various deforming heights $(H)$ of the downscaled specimen with different grain sizes.

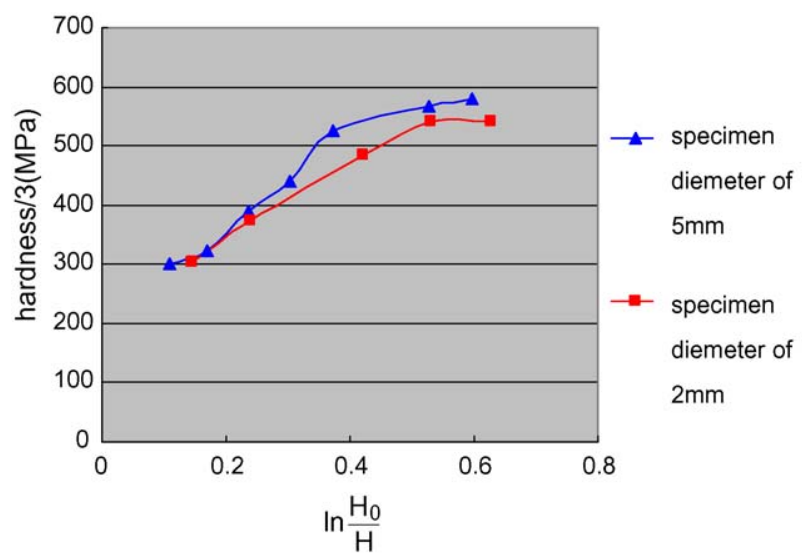

Fig. 6. Measured hardness at various deforming heights $(H)$ of specimens with different diameters (grain size $=29 \mu \mathrm{m}$ ).

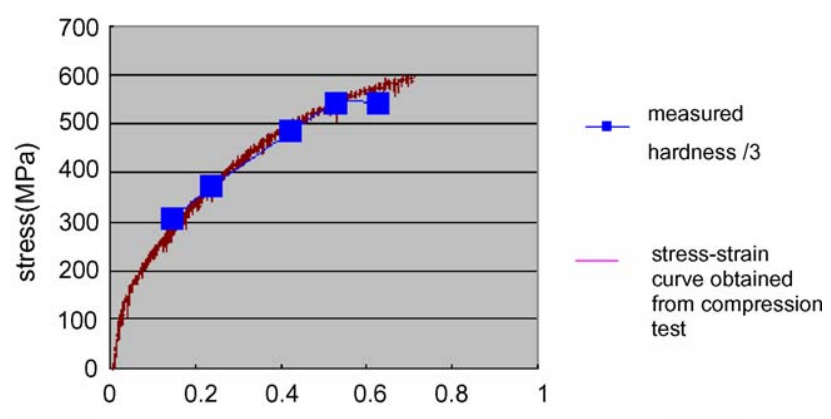

Fig. 7. Comparison of hardness and flow stress at various strains for the downscaled specimen with grain size of $29 \mu \mathrm{m}$.

specimen, which is similar to that observed in the stress-strain relations obtained in the compression tests, and the size effect is clearly noted. It is thus concluded that the size effect is also present in the material hardness.

In order to examine if the theoretical proportionality between the hardness and the flow stress is still valid in a specimen with downscaled dimensions, the values of hardness divided by three (hardness/3) are compared with the stress-strain relations obtained in the compression tests. Fig. 7 displays the comparison for the downscaled specimen with grain size of $29 \mu \mathrm{m}$. It is seen in Fig. 7 that the values of hardness/ 3 agree very well with the stress-strain curve up to the strain about 0.5 and then an insignificant discrepancy appears at higher strains. It indicates that the theoretical proportionality between the hardness and the flow stress still exists in a specimen with downscaled dimensions. It therefore implies that the stress-strain curve can be constructed from the hardness measurement if the accurate data acquisition is not available in the micro-forming test equipment.

\section{Concluding remarks}

The compression test results reveal that the flow stress becomes smaller when the specimen is downscaled to a small size. The grain size also affects the flow stress, but the difference is not significant for the range of the grain size examined. 
The finite element simulations performed in the present study also indicate that the proper position for the hardness measurement is located at 0.875 of the radius away from the center. The size effect on the material hardness is clearly observed in the micro-hardness tests. The hardness decreases as the specimen is downscaled to a small size, which is similar to the size effect appeared in the stress-strain relations. Thus, the size effect on the material properties in the micro-forming process is confirmed. However, the deformation mechanism causing the size effect is yet to be examined, though some models have been proposed to date. The good agreement between the measured hardness and the flow stress confirms that the theoretical proportionality between the hardness and the flow stress is still valid in a specimen with downscaled dimensions. It further suggests that the stress-strain relations of a downsized specimen can be constructed from the micro-hardness tests in conjunction with the compression tests, if the accurate data acquisition is difficult to be achieved in the compression test equipment for a downscaled specimen.

\section{Acknowledgements}

The authors would like to thank the National Science Council of the Republic of China for financially supporting this research under Contract No. NSC 92-2212-E-002-076, which makes the experimental work possible. They are also grateful to SFTC for the help in running the DEFORM-3D software.

\section{References}

[1] M. Geiger, M. Kleiner, R. Eckstein, N. Tiesier, U. Engel, Microforming, Ann. CIRP 2 (2001) 445.

[2] T.A. Kals, R. Eckstein, Miniaturization in sheet metal working, J. Mater. Process. Technol. 103 (2000) 95-101.

[3] X. Yao, S. Zajac, B. Hutchinson, Estimation of compression flow stress from post-deformation hardness in Al-Mg alloys, Script. Mater. 41 (3) (1999) 253-258.

[4] J.H. Ahn, E.C. Jeon, Y. Choi, Y.H. Lee, D. Kwon, Derivation of tensile flow properties of thin films using nanoidentation technique, Curr. Appl. Phys. 2 (2002) 525-531. 